\title{
Review of: "Activation of transient receptor potential vanilloid 4 protects articular cartilage against inflammatory responses via CaMKK/AMPK/NF-KB signaling pathway"
}

Kang Xu

Potential competing interests: The author(s) declared that no potential competing interests exist.

The manuscript submitted by Nobunori et al describes the phenomenon, TRPV4 activation had chondroprotective effects on articular cartilage stimulated with IL-1 $\beta$ by activating CaMKK/AMPK and suppressing the NF-KB pathway. This manuscript firstly demonstrated that TRPV4 activation inhibits NF-KB phosphorylation by activating the CaMKK/AMPK pathway in chondrocytes in the presence of IL-1 $\beta$, resulting in a chondroprotective effect, but some results are not rigorous.

1. Authors need to introduce the role of IL-1 $\beta$ in OA and the mechanisms of OA more detail.

2. The MTS assays just using 100, 1000 and 10000 pM of GSK101, the number of detected concentrations is too small that lacks persuasiveness.

3. In Fig.1b, the in vitro assay to detect the optimization of GSK101 just detecting the expression of mRNA, such as MMP-13, AGC and SOX9, this is also not convinced.

4. Why the treatment times of GSK101 to chondrocytes in the in vitro assays were not uniform? Some of them was $12 \mathrm{~h}$ and $48 \mathrm{~h}$, some even $30 \mathrm{~min}$.

5. The cartilage explant cultures just using bovine articular cartilage. Why not used the human articular cartilage sample instead? As we know the clinical samples can be collected from OA patient. Also, figure $2 \mathrm{~d}$, please indicate the location of the injured site.

6. In Fig.3, there is no mark " $\int$ " in the picture, but it does in the legend.

7. In Fig.4a, Real-time PCR analysis of relative expression of MMP-13, AGC and SOX9 in BACs treated with IL-1ß, GSK101, and STO-609 for $12 \mathrm{~h}$. This assay just detecting the mRNA level of MMP-13, AGC and SOX9, without the protein level. Besides, in Fig.4b and c, the Real-time PCR and Western Blot assays just detecting the expression of MMP-13, this is also not convinced.

8. Activation of TRPV4 protects articular cartilage against inflammatory responses via CaMKK/AMPK/NF-KB signaling pathway, but the role of TRPV4 on inflammation had not been studied. More mechanistic studies or description about it in the discussion should be performed.

9. The authors need to provide Animal Ethics Certificate number in the Methods.

10. Methods section need to explain better. 
